\title{
8.2 PULSAR MODELS AND RADIATION PROPERTIES
}

\author{
V. RADHAKRISHNAN \\ Division of Radiophysics, CSIRO, P.O. Box 76, Epping, N.S.W., Australia
}

\begin{abstract}
Dr. Radhakrishnan outlined a geometrical theory of a rotating pattern of field lines, which would account for the pattern of polarisation observed in the integrated radio pulses from pulsars.
\end{abstract}

The following summary has been prepared by the Editors.

Any theory for the radiation mechanism in pulsars requires a description of the properties of the region in terms of brightness temperatures, polarisation, spectrum, and directivity. There is a close relation between the observed polarisation properties and the directivity, since the sweep of position angle within the pulses is independent of frequency and must be a purely geometrical effect related to rotation. Since the position angle can change rapidly through a large range, the radiation must come from a pole, presumably a magnetic pole. The source of the radiation must be well within the velocity of light cylinder, since the field lines must be close to a dipole configuration.

Other so-called intrinsic properties of the radiation not accounted for by the above model are rapid pulse-to-pulse variations, variable circular polarization, and marching subpulses. All of these properties are more pronounced at lower radio frequencies. This suggests strongly an origin in modulation or propagation phenomena. It is hypothesized that all of the above variable characteristics are imposed on the radiation in the circumstellar region by a circulating diffracting screen permeated by a moderately strong magnetic field.

\section{Discussion}

F. G. Smith: You suggest that the sweep of polarization angle implies that the source is seen near the pole of a magnetic field. This is not necessarily so. The sweep of angle is an intrinsic part of my model which allows the source to be seen from any angle, including from the equator. Secondly, I again emphasize that if you are to obtain circular polarization by the propagation effect then you must take care that this effect is not frequency selective. Further, you must make the polarization change during a single pulse just sufficiently for one complete change of Stokes parameters to occur, no more and no less.

J.M. Cohen: If the Goldreich-Julian procedure is repeated for an oblique rotator, it turns out that the radiation zone continues right up to the surface of the star (in some sense). It is necessary to use the full radiation solution in order to match the boundary conditions of the neutron star surface. A near-field approximation does not work.

J.P. Ostriker: If the radiation is attributed to bunching of electrons, then there is no frequency dependence. However there might be some screening which is frequency dependent.

$F$. D. Drake: The picture of pulsar emission as radiation from coherent bunches of particles at radio frequencies, and ordinary synchrotron radiation from the same particles individually producing the optical and higher frequencies, can explain pulsar variability. For the radio emission we depend upon the construction of a relatively small number of bunches of particles which vary in size. There can be large statistical variations in these leading to large radio variations. However since the particles radiate at optical frequencies independently, there would be no significant variation. 
V. Radhakrishnan: I can suggest an observational test of this idea. It implies that at higher frequencies the mean polarization will approach that observed optically. In particular, circular polarization will decrease and the marching sub-pulses should disappear. The detailed structure of the pulse will, in general, become smoothed out.

F.C. Michel: By this model one would expect the pulse intensity to be flat at maximum when the line of sight travels very close to the pole. However in the case of the Crab pulsar, the maximum appears to be sharp at all resolutions.

V. Radhakrishnan: The model is primarily intended to explain the pattern of polarization, but this does not exclude the possibility of obtaining almost any pulse shape as the line of sight sweeps across the emitting region. 